\title{
An Overview on Different Processing Parameters in Particulate Reinforced Metal Matrix Composite Fabricated by Stir Casting Process
}

\author{
Bagesh Bihari*, Anil Kumar Singh** \\ *Research Scholar, Department of Foundry Technology, National Institute of Foundry and Forge Technology, \\ Hatia, Ranchi, India \\ ** Professor, Department of Foundry Technology, National Institute of Foundry and Forge Technology, Hatia, \\ Ranchi, India
}

\begin{abstract}
In the past few years the global need for reduced weight, low cost, high wear resistance and good quality materials has caused a shift in research from monolith ic to composite materials. Metal Matrix Composites (MMCs) have evoked a keen interest in recent times for potential applications in marine, aerospace and automotive industries owing to their superior strength to weight ratio and high temperature resistance. Driving force for the utilization of AMCs include performance, economic and environmental benefits. The widespread adoption of particulate metal matrix composites for engineering applications has been hindered by the high cost of producing components. Achieving a uniform distribution of reinforcement with in the matrix is one such challenge which impacts directly on the properties and quality of the composite material. In the case of particle reinforced metal matrix composites, the distribution of the reinforcement particles in the matrix alloy is influenced by several factors during casting. These include the rheological behaviour of the matrix melt, the particle incorporation method, interactions of the particles and the matrix before, during and after mixing, and the changing particle distribution during solidification. In this paper, research relevant to these factors which influences the particle distribution is reviewed and discussed in order to achieve a good distribution of reinforcement partic les in a cast metal matrix composite (MMC).
\end{abstract}

Keywords: Metal Matrix Composite, Process Parameter, Stir Casting

\section{I.INTRODUCTION}

Metal matrix composites (MMCs) are now recognized as important engineering and structural materials for application in aerospace, construction, automotive and leisure industries. The reinforcement of light weight aluminium alloys with short fibers, platelets and particle of ceramics such as silicon carbide or alumina results in composite of high specific strength and stiffness suitable for engineering applications like marine, aerospace and automotive [1-9].

High demands on material for better overall performance has led to extensive research and development efforts in the composites fields. Among the composites field, the aluminium based metal matrix composite materials are widely used. To meet emerging need, innovations in materials processing enabled achieving an enhancement in stiffness, realization of high strength to weight ratio, an improvement in wear resistance, maintaining strength at elevated temperatures $[12,13]$.

MMCs' attributes include alterations in mechanical behaviour (e.g., tensile and compressive properties, creep, notch resistance, and tribology) and physical properties (e.g., intermediate density, thermal expansion, and thermal diffusivity) by the reinforced phase. The limitations of materials are thermal fatigue, thermo chemical compatibility, and low-transverse creep resistance. Most of the research work has been dealing with aluminium matrix. This is because aluminium is lighter weight which is first requirement in most of the industries. As a result, these materials are now being rapidly utilized in industries that traditionally used metals, and these have become the forefront of research and development activity in the many related areas $[14,15]$.

There are several routes by which the reinforcement may be introduced in the matrix. The stir casting technique is an effective method for preparation of light weight MMC [10,11].The foundry casting processes have been a favoured processing method as they lend themselves to the manufacture of large number of complex shaped components. Especially, the stir casting mostly used to produce the Particulate reinforced metal matrix composite. The major problem in this technology is to obtain sufficient wetting of particle by the liquid metal and to get a homogeneous 
dispersion of the ceramic particles [16]. Several structural defects such as porosity, particle clustering, oxide inclusions and interfacial reactions were found to arise from the unsatisfactory casting technology [17].

\section{STIR CASTING IN BRIEF}

Stir casting of metal matrix composites was initiated in 1968, when S. Ray introduced alumina particles into aluminium melt by stirring molten aluminium alloys containing the ceramic powders [18]. A Schematic view of set up for preparation of composites through stir casting process is illustrated in Fig. 1 [19].

In a stir casting process, the reinforcing phases (usually in powder form) are distributed into molten aluminium by mechanical stirring. Mechanical stirring in the furnace is a key element of this process. The resultant molten alloy, with ceramic particles, can then be used for die casting, permanent mould casting, or sand casting [20,21].

A major problem associated with the stir casting process is the segregation of reinforcing particles which is caused by the surfacing or settling of the reinforcement particles during the melting and casting processes. The final distribution of the particles in the solid depends on material properties and process parameters such as the wetting condition of the particles with the melt, strength of mixing, relative density and rate of solidification. The distribution of the particles in the molten matrix depends on the geometry of the mechanical stirrer, stirring parameters, placement of the mechanical stirrer in the melt, melting temperature, and the characteristics of the particles added [22,23].

An interesting recent development in stir casting is a two-step mixing process [24]. In this process, the matrix material is heated to above its liquidus temperature so that the metal is totally melted. The melt is then cooled down to a temperature between the liquidus and solidus points and kept in a semi-solid state. At this stage, the preheated particles are added and mixed. The slurry is again heated to a fully liquid state and mixed thoroughly.

The effectiveness of this two-step processing method is mainly attributed to its ability to break the gas layer around the particle surface. Particles usually have a thin layer of gas absorbed on their surface, which impedes wetting between the particles and molten metal. Compared with conventional stirring, the mixing of the particles in the semi-solid state can more effectively break the gas layer because the high melt $\mathrm{v}$ iscosity produces a more abrasive action on the particle surface. Hence, the breaking of the gas layer improves the effectiveness of the subsequent mixing in a fully liquid state. Stir casting allows for the use of conventional metal processing methods with the addition of an appropriate stirring system such as mechanical stirring; ultrasonic or electromagnetic stirring; or centrifugal force stirring [25]. The major advantage of stir casting is its applicability to large quantity production. Among all the wellestablished metal matrix composite fabrication methods, stir casting is the most economical (Compared to other methods, stir casting costs as little as one third to one tenth for mass production $[26,27]$ For that reason, stir casting is currently the most popular commercial method of producing alu min iu m based composites.

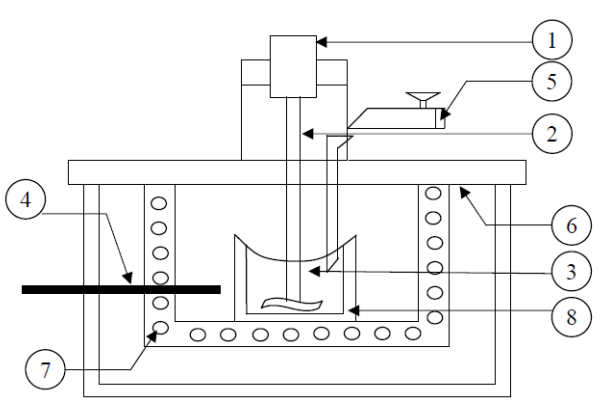

Fig. 1. Schematic view of setup for fabrication of

1. Motor, composite

2. Shaft,

3. Molten aluminium,

4. Thermocouple,

5. Particle injection chamber

6. Insulation hard board

7. Furnace

8. Graphite crucible

\section{PROCESS PARAMETER}

For manufacturing of composite material by stir casting, knowledge of its operating parameter are very essential. As there is various process parameters if they properly controlled can lead to the improved characteristic in composite material.

\section{STIRRER DES IGN}

Stirrer design is an important factor in stir casting process as it is responsible for vortex formation and uniform distribution of reinforcements. Different designs and material for designing stirrers are available but stirrer made out of graphite is more durable compared to metallic stirrers. For better machining of stirrer, fine grain graphite material with control machining is required. Graphite stirrer is generally used in the shape of flat blade due to the difficulty in machining. Hence a graphite turbine stirrer has been designed which is more durable and effective in particle dispersion compared to other stirrers. It 
is experimentally found that steel stirrer gets damaged only after 1-2 melting process where as graphite stirrer lasts up to $12-13$ melting.

\section{STIRRER POSITION}

It has been experimentally found that effective vortex is formed at $700 \mathrm{rpm}$ when the stirrer is at $1 / 3$ of the melt height from the bottom. It is observed that if the height is more than $1 / 3$ of the melt height from the bottom, a small ineffective vortex is formed, which results in insufficient dispersion of reinforcement particles. Also, if the height is less than $1 / 3$ of the melt height from the bottom, the vortex disappears and particle dispersion is stopped. According to the experimental observation the stirrer should be placed at a height which is no more than $30 \%$ from the base to avoid accumulation of particles at the bottom of the mixture.

\section{BLADE ANGLE}

The blade angle and number of blades are prominent factor which decides the flow pattern of the liquid metal at the time of stirring. The blade with angle $45^{\circ} \& 60^{\circ}$ will give the uniform distribution. The number of blade should be 4 . Blade should be $20 \mathrm{~mm}$ above the bottom of the crucible [30]. Blade pattern drastically affect the flow pattern.

\section{MELT TEMPERATURE}

It is an important process parameter. It is related to the melting temperature of matrix i.e. aluminium. The processing temperature is mainly influence the viscosity of Al matrix. The change of viscosity influences the particle distribution in the matrix. The viscosity of liquid decreased when increasing processing temperature with increasing stirring time [30]. It also accelerates the chemical reaction b/w matrix and reinforcement. Melt temperature is kept at $780-800^{\circ} \mathrm{C}$ as the increase in reinforcement particles amount increase the melt viscosity, which hinder stirring speed. It is experimentally found that the melt temperature of $780-800^{\circ} \mathrm{C}$ is suitable for controlling the stirring speed and effective dispersion. Moreover, the optimum temperature of Al-alloy melt for the dispersion of particles is found to be in the range of $740-800^{\circ} \mathrm{C}$ [Ban 1983]. As the temperature falls below $700^{\circ} \mathrm{C}$, viscosity of the melt increases and hence after the addition of reinforcement particles viscosity increases too much and results in the solidification of melt. If the temperature is kept above $800^{\circ} \mathrm{C}$ the viscosity of melt reduces too much that result in the settling of particles in the melt without dispersing in the melt during stirring. The changes of viscosity influence the particle distribution in the Al matrix.
At higher viscosity with lower temperature (below $800^{\circ} \mathrm{C}$ ), the geometric contacts of particles is restricted by vortex of molten metal. Therefore, the particles are distributed uniformly in this range. But, at lower viscosity with higher temperature (above $800^{\circ} \mathrm{C}$ ), the geometric of particles are captured and it was unable to restricts by the vortex of molten metal under the constant stirring speed. The conclusion can be drawn that viscosity of liquid de-creased when increasing processing temperatures with increasing holding time.

\subsection{EFFECT OF MELT TEMPERATURES}

The melt temperature is mainly influencing the change in viscosity of Al matrix and also it accelerating the chemical reactions between them. This change of viscosity was calculated theoretically by using Arrhenius equation [33].

$$
\eta=\eta_{0} \mathrm{e}^{-\mathrm{E} /(\mathrm{RT})}
$$

where $\eta_{0}$-viscosity of aluminium at the melting temperature, E-Activation energy for viscous flow of aluminium, R-Universal gas constant, T-Melt temperature.

\section{PARTICLE PREHEATING TEMPERATURE}

Particle preheating temperature is very necessary to remove moisture and gases from the surface of the particulates. If moisture and gases will be present then the chances of agglomeration will be more due to presence of entrapped air in the particle. Preheating of the particles before dispersion into the melt aids their transfer by causing desorption of adsorbed gases from the particle surface. Preheating should be done at $800^{\circ} \mathrm{c}$ for $2 \mathrm{hrs}$ which assists in removing surface impurities and in desorption of gasses and alters the surface composition by forming an oxide layer on the surface.

Hence a clean surface provides a better opportunity for melt particles interaction and thus promotes the wettability of reinforcement with the matrix. Thus results in strong interfacial bonding [31].

\section{PARTICLE SIZE AND AMOUNT}

It is observed that particle size and amount affects the manufacturing process of composite. When the particle size is very less $(<20 \mu \mathrm{m})$, it results in agglomeration either at the bottom or top of melt in the crucible. It is observed that if particle size is too much small, particles tends to float on the surface of melt. Fine particles have a tendency to agglomerate during synthesis, when added above a certain amount, depending upon its size. These agglomerates entrap air and there is a decrease in their density. A combined effect of decrease in 
density, buoyant force of the melt and stirring action results in a floatation of these agglomerates on liquid melt during synthesis. Larger particle size $(>200 \mu \mathrm{m})$ results in the non dispersion of particles and settling of particles at the bottom of crucible. It is observed that particles are rejected from the melt when the added amount is more than $10 \mathrm{wt} \%$.

\section{PARTICLE INCORPORATION RATE}

It is observed from the experimental data that a good dispersion is achieved when PIR is less (10-20 $\mathrm{gm} / \mathrm{min})$. Dispersion of particles reduces significantly when PIR is $30-40 \mathrm{~g} \mathrm{~m} / \mathrm{min}$. High PIR leads to agglomeration of particles followed by rejection from the alloy melt. It is observed that there is no dispersion of particles for PIR above 40 $\mathrm{gm} / \mathrm{min}$. In such high rate $(>40 \mathrm{~g} \mathrm{~m} / \mathrm{min})$ particles settles at the bottom of the crucible without getting wetted by the liquid alloy. The decrease in particle dispersion with increased PIR is due to the agglomeration of the particles at the top of the melt. The agglomerates are difficult to wet by aluminium melt and hence they tend to settle at the bottom. During particle incorporation, particle free fall in the melt is more important so that particles can disperse uniformly in the melt. To have a good quality of casting the particle incorporation rate must be uniform. If it is non-uniform it promotes clustering of particles at some places which in turn enhances the porosity defect and inclusion defect, so the feed rate of particles must be uniform.

Dispersion of particles in Al-alloy for different Particle Incorporation Rate (PIR):

\begin{tabular}{|l|l|}
\hline $\begin{array}{l}\text { Particle } \\
\text { incorporation rate } \\
\text { (gram/minute) }\end{array}$ & Observation \\
\hline $10-20$ & Good dispersion \\
\hline $20-40$ & $\begin{array}{l}\text { Poor } \\
\text { dispersion/Agglomerati } \\
\text { on }\end{array}$ \\
\hline More than 40 & Particle rejection \\
\hline
\end{tabular}

\section{STIRRING SPEED}

Stirring speed is the important process parameter as stirring is necessary to help in promoting wettability i.e. bonding between matrix $\&$ reinforcement. The control of speed is very important for successful production of casting. Stirring speed also influences the structure, the most common effect of increase in speed being to promote refinement and instability of the liquid mass at very low speed. It is logical to use the highest speed consistent with the avoidance of tearing [28].

Stirring speed will directly control the flow pattern of the molten metal. Parallel flow will not promote good reinforcement mixing with the matrix. Hence flow pattern should be controlled turbulence flow. Pattern of flow from inward to outward direction is best. [30].

A mechanical force can usually be used to overcome surface tension to improve wettability. The stirring speed has been raised up to a constant speed of $700 \mathrm{rpm}$ as it is the maximum speed in which smooth vortex is formed without melt turbulence and spattering. It is experimentally found that when stirring speed is less than $700 \mathrm{rpm}$, ineffective vortex is formed that leads to non dispersion of particles in the aluminium melt. When the stirring speed is raised to $800 \mathrm{rpm}$, there is turbulence and spattering of melt.

\section{STIRRING TIME}

Stirring promotes uniform distribution of the particles in the liquid and to create perfect interface bond $b / w$ reinforcement and matrix. The stirring time $\mathrm{b} / \mathrm{w}$ matrix and reinforcement is considered as important factor in the processing of composite. For uniform distribution of reinforcement in matrix in metal flow pattern should from outward to inward. Stirring time depends upon the amount of reinforcement particles to be dispersed in aluminium alloy melt.

\subsection{EFFECT OF STIRRING TIME}

Effect of the stirring time helps in the fabrication of MMC by two ways: to distribute the particles in the liquid, and to create perfect interface bond between reinforcement and matrix. When the stirring time is 10 minutes, the particles are distributed uniformly in the matrix at $800^{\circ} \mathrm{C}$. The liquid matrix has sufficient viscosity in the temperature range, and velocity of particles flow is small. In the case of 20 minutes stirring time, the liquid has sufficient viscosity at lower temperature $\left(<800^{\circ} \mathrm{C}\right)$ but the contacts time between matrix and reinforcement too large. During this period, the particles are distributed uniformly in liquid even though some of particles form cluster which could be also are located in the matrix region. A vortex created during the stirring can suck the air or gas bubbles in to the liquid metal. As the results, the particles were attached with air bubbles to form the particles cluster in the matrix.

\section{ADDITION OF MAGNESIUM}

Addition of Magnesium enhances the wettability. The basic means used to improve wetting are: (a) Increasing the surface energy of the solid, (b) Decreasing the surface tension of the liquid matrix alloy \& (c) Decreasing the solidliquid interfacial energy at the particle-matrix. However increase the content above 1wt. \% increases viscosity of slurry and hence uniform particle distribution will be difficult [32]. 


\section{DEGASS ING}

As aluminium melts it starts to react with environment oxygen and will produce an oxide layer at the top. This oxide layer will avoid further oxidation but along that it will difficult to brake. So such layer will be big trouble for reinforcement mixture with metal. So in order to avoid this Hexachloroethane $\left(\mathrm{C}_{2} \mathrm{Cl}_{6}-0.3 \%\right)$ is added for the degassing purpose.

\section{MOULD TEMPERATURE}

In casting porosity is the prime defect. In order to avoid these preheating the permanent mould is good solution. It will help in removing the entrapped gases from the slurry in mould It will also enhance the mechanical properties of the cast AMC. The use of metallic mould produces marked refinement when compared with sand mould but mould temperature is only of secondary importance in relation to the structure formation. Its principal signification lies in the degree of expansion of the mould with preheating. Expansion diminishes the risk of tearing in casting. In nonferrous castings, the mould temperature should neither be too low or too high. The metallic mould should be at least 25 $\mathrm{mm}$ thick with the thickness increasing with size and weight of casting [29].

\section{MOULD COATINGS}

Various types of coating materials are used. The coating material is sprayed on the inside of the metal mould. The purpose of the coating is to reduce the heat transfer to the mould. Defects like shrinkage and cracking that are likely to occur in metal moulds can be eliminated, thus increasing the die life. The role of coating and solidification can be adjusted to the optimum value for a particular alloy by varying the thickness of coating layer. For aluminium alloys, the coating is a mixture of Silicate and graphite in water.

\section{MOULD LIFE}

Metal mould in casting is subjected to thermal stresses due to continuous operation. This may lead to failure of the mould. The magnitude of the stresses depends on the mould thickness and thickness of the coating layer, both of which influence the production rate. Deterioration takes place faster in cast iron mould than in steel mould [29].

\section{POURING TEMPERATURE}

Pouring temperature exerts a major role on the mode of solidification and needs to determine partly in relation to type of structure required. Low temperature is associated with maximum grain refinement and equiaxed structures while higher temperature promotes columnar growth in many alloys. However practical consideration limits the range. The pouring temperature must be sufficiently high to ensure satisfactory metal flow and freedom from cold laps whilst avoiding coarse structures [29].

\section{POURING RATE}

This is governed primarily by the need to finish casting before the metal become sluggish. Although too high a rate can cause excessive turbulence and rejection. In practice slow pouring offers number advantages. Directional solidification and feeding are promoted whilst the slow development of full centrifugal pressure on the other solidification skin reduces and risk of tearing. Excessive slow pouring rate and low pouring temperature would lead to form surface lap [29].

\section{CONCLUSION}

In the present investigation the aim is to study the various operating parameter of stir casting process. While manufacturing aluminium metal matrix composite we come to know that process parameter plays a major role for uniform distribution of reinforcement. The following conclusion can be observed based on above investigations:

1) For uniform dispersion of material blade angle should be $45^{\circ}$ or $60^{\circ}$ and no of blade should be 4. Stirrer position is the most important parameter for the vortex formation.

2) For good wettability we need to keep operating temperature at $780-800^{\circ} \mathrm{c}$, stirring speed should be $700 \mathrm{rpm}$, stirring time should varies according to amount of reinforcement and PIR.

3) Preheating of reinforcement particle is much more essential to improve wettability and preheating of mould helps in reducing porosity as well as increases mechanical properties.

\section{REFERENCES}

[1]. Subrat Ray, Expanding Frontier of Metallic Materials for Structural applications, IIM Metal News, Vol.12, No. 4, August 2009.

[2]. P.K Rohatgi, Metal-matrix Composites, Defense Science Journal, Vol.43, No 4, October 1993, pp 323-349.

[3]. B.P. Samal, A.K. Mishra, S.C.Panigrahi, S.C.Mishra, Plunger Technique: A New Approach to Stir Casting AMMC Preparation, Accepted paper in Journal of Materials and Metallurgical Engineering, 2013.

[4]. B.P. Samal, A.K. Misra S.C.Panigrahi, S.C.Mishra, A novel technique for improved recovery of Mg-Analysis of the Microstructure and Physical properties, 
Journal of Materials and Metallurgical Engineering, Volume 3, Issue 1(2013), 1-7.

[5]. J.Hashim, L.Looney, M.S.J.Hashmi, Metal Matrix Composites: Production by the stir casting method, Journal of Materials Processing Tech.92-93 (1999), 1-7.

[6]. P. Rohatagi, Cast metal matrix composites: Past, present and future, AFS Trans 2001:01-133.

[7]. J. Hashim, The production of cast metal matrix composite by a modified stir casting method, Journal Technology, 35(A) Dis.2001: 9-20.

[8]. M. Ramachandra and K. Radhakrishna, Study of Abrasive wear behavior of Al-Si (12\%) SiC metal matrix composite synthesized using vortex method, International Symposium of Research Students on Materials Science and Engineering, December-20-22, 2004, Chennai, India.

[9]. A.Wlodarczyk-flig ier, L.A.Dobrzanski, M. Adamiak, Manufacturing of aluminium matrix composite materials reinforced by $\mathrm{Al}_{2} \mathrm{O}_{3}$ particles, Journal of Achievements in Materials and Manufacturing Engineering. Vol- 27 Issue 1 March 2008, 99-102.

[10]. T. P. Rajan, R. M. Pillai and B. C. Pai, Review Reinforcement Coatings and Interfaces in Aluminium Metal Matrix Composites, Journal Material Science, Vol. 33, No. 14, 1998, pp. 3491-3503.

[11]. M. Jayamathi, S. Seshan, S. V. Kailas, K. Kumar and T. S. Srivatsan, Influence of Reinforcement on Microstructure and Mechanical Response of a Magnesium Alloy, Current Science, Vol. 87, No. 9, 2004, pp. 1218-1231.

[12]. V. K. Lindroos and M. J. Talvitie, Recent Advances in Metal Matrix Composites, Journal of Material Processing Technology, Vol. 53, 1995, pp. 273-284.

[13]. L. M. Tham, M. Gupta and L. Cheng, Effect of Limited Matrix-Rein forcement Interfacial Reaction on Enhancing the Mechanical Properties of Aluminium-Silicon Carbide Composites, Acta Materialia, Vol. 49, No. 16, 2001, pp. 3243-3253.

[14]. S. Sarkar, S.Sen \& S.C Mishra, M.K Kudelwar \& S. Mohan, Studies on Aluminium-Fly- Ash Composite Produced by Impeller Mixing, Journal of Reinforced Plastics and Composites, Vol.00, No-00 / 2008, 1-6.

[15]. A.R. Kennedy, A. E. Karantzalis, S. M. Wyatt, The microstructure and mechanical properties of $\mathrm{TiC}$ and $\mathrm{TiB}_{2}$ reinforced cast metal matrix composites, journal of materials science 34(1999)933-940.

[16]. J. Hashim, L. Looney and M. S. J. Hashmi, Metal Matrix Composites: Production by the Stir Casting Method, Journal of Materials Processing Technology, Vol. 119, No. 1-3, 1999, pp. 329-335

[17]. W. Zhou and Z. M. Xu, Casting of SiC Reinforced Metal Matrix Composites, Journal of Materials Processing Technology, Vol. 63, No. 1-3, 1997, pp. 358363.

[18]. S. Ray, M.Tech Dissertation, Indian Institute of Technology, Kanpur, 1969.

[19]. A. Luo, Metall. Mater. Trans. A 26A (1995) 2445.

[20]. A. Luo, Metall. Mater. Trans. A 26A (1995) 2445.

[21]. R. A. Saravanan and M. K. Surappa, Material Science Enineering, A 276 (2000) 108.

[22]. N. Harnby, M.F.Edward and A.W.Nienow, Mixing in Process Industries (Butterworths, London, 1985).

[23]. F. A. Girot, L. Albingre, J. M. Quenisset and R. Naslain, J. Met. 39 (1987).

[24]. P. Rohatgi, Modern Casting, April (1988) 47.

[25]. M.K. Surappa, Journal of Material Processing and Technology, 63 (1997) 325.

[26]. D. M. Skibo, D. M. Schuster and L. Jolla, US Patent No. 4786467 (1988).

[27]. Sanjeev Das, V. Udhayabanu, S. Das, K. Das, Synthesis and characterization of Zircon Sand/A1-4.5 wt\% $\mathrm{Cu}$ Composite produced by Stir Casting Route, Journal of Material Science, (2006) 41:4668-4677.

[28]. T. P. D. Rajan, R.M. Pillai, B.C. Pai, K.G. Satyanarayana, P.K. Rohatgi, Fabrication and characterisation of $\mathrm{Al}-7 \mathrm{Si}-0.35 \mathrm{Mg} / \mathrm{fly}$ ash metal matrix composites processed by different stir casting routes, Composites Science and Technology 67 (2007) 33693377.

[29]. S. Naher, D. Brabazon, L. Looney, Simulation of the Stir Casting Process, Journal of Materials Processing Technology 143-144 (2003) 567-571

[30]. Pradeep Sharma, Gulshan Chauhan, Neeraj Sharma, Production of Amc By Stir CastingAn Overview, International Journal of Contemporary Practises Vol.2 Issue1.

[31]. Dunia Abdul Saheb, Aluminum Silicon Carbide and Aluminum Graphite Pariculate Composites, ARPN Journal of Engineering and Applied Sciences Vol. 6, No.10, October 2011, ISSN:1819-6608 
[32]. F. Miani and P. Matteazzi, Estimation of Viscosity in under Cooled Liquid Metal Alloys, Journal of Non-Crystalline Solids, Vol. 143, 1992, pp. 140-146. 